International Journal of Theoretical and Applied Finance

Vol. 14, No. 8 (2011) 1385-1387

(C) World Scientific Publishing Company

DOI: $10.1142 / \mathrm{S} 0219024911006887$

\title{
Author Index Volume 14 (2011)
}

Albanese, C., Kernel Conver-
gence Estimates for Dif-
fusions with Continuous
Coefficients

Asvanunt, A., Broadie, M. \& Sundaresan, S., Managing Corporate Liquidity: Strategies and Pricing Implications

Badescu, A., Elliott, R. J., Kulperger, R., Miettinen, J. \& Siu, T. K., A Comparison of Pricing Kernels for GARCH Option Pricing with Generalized Hyperbolic Distributions

Bo, L., Wang, Y. \& Yang, X., Derivative Pricing Based on the Exchange Rate in a Target Zone with Realignment

Boyarchenko, M. \& Boyarchenko, S., Double Barrier Options in Regime-Switching Hyper-Exponential JumpDiffusion Models

Boyarchenko, M., de Innocentis, M. \& Levendorskii, S., Prices of Barrier and FirstTouch Digital Options in Lévy-Driven Models, Near Barrier

Boyarchenko, S., see Boyarchenko, M.

Brigo, D., Pallavicini, A. \& Papatheodorou, V., Arbitrage-Free Valuation of Bilateral Counterparty Risk for Interest-Rate Products: Impact of Volatilities and Correlations

Broadie, M., see Asvanunt, A.

Brodén, M. \& Tankov, P., Tracking Errors from Discrete Hedging in Exponential Lévy Models
Carmona, R. \& Nadtochiy, S., Tangent Models as a Mathematical Framework for Dynamic Calibration

Carr, P., Semi-Static Hedging of Barrier Options Under Poisson Jumps

Carr, P., Zhang, H. \& Hadjiliadis, O., Maximum Drawdown Insurance

Carton de Wiart, B. \& Dempster, M. A. H., Wavelet Optimized Valuation of Financial Derivatives

Chen, W.-T., see Zhu, S.-P.

5 (2011) $669 \quad$ Cheridito, P. \& Kupper, M., Composition of TimeConsistent Dynamic Monetary Risk Measures in Discrete Time

6 (2011) $945 \quad$ D'Amico, G., Janssen, J. \& Manca, R., A NonHomogeneous SemiMarkov Reward Model for the Credit Spread Computation

7 (2011) 1005 Da Fonseca, J., Grasselli, M. \& Ielpo, F., Hedging (Co)Variance Risk with Variance Swaps

de Innocentis, M., see Boyarchenko, M.

7 (2011) $1045 \quad$ Dempster, M. A. H., see Carton de Wiart, B.

7 (2011) $1005 \quad$ Dimitroff, G., Lorenz, S. \& Szimayer, A., A Parsimonious Multi-Asset Heston Model: Calibration and Derivative Pricing

1 (2011) 107

7 (2011) 1091

8 (2011) 1195

7 (2011) 1113

8 (2011) 1279

1 (2011) 137

2 (2011) 221

6 (2011) 899

7 (2011) 1045

7 (2011) 1113

6 (2011) 773

3 (2011) 369

6 (2011) 803
Dmitrašinović-Vidović,

G., Lari-Lavassani, A., Li, X. \& Ware, A., Dynamic Portfolio Selection Under Capital-atRisk with no Short-Selling Constraints

Dokuchaev, N., Option Pricing via Maximization Over 
Uncertainty and Correction of Volatility Smile

Elliott, R. J., see Badescu, A. Eriksson, B. \& Pistorius, M., Method of Moments Approach to Pricing Double Barrier Contracts in Polynomial JumpDiffusion Models

Forde, M., Exact Pricing and Large-Time Asymptotics for the Modified SABR Model and the Brownian Exponential Functional

Fries, C. P. \& Joshi, M. S., Perturbation Stable Conditional Analytic MonteCarlo Pricing Scheme for Auto-Callable Products

Frittelli, M. \& Maggis, M., Conditional Certainty Equivalent

Frittelli, M. \& Rosazza Gianin, E., On the Penalty Function and on Continuity Properties of Risk Measures

Föllmer, H. \& Penner, I., Monetary Valuation of Cash Flows Under Knightian Uncertainty

Fukasawa, M., Ishida, I., Maghrebi, N., Oya, K. Ubukata, M. \& Yamazaki, K., Model-Free Implied Volatility: From Surface to Index

Futami, H., Regime Switching Term Structure Model Under Partial Information

Gassiat, P., Pham, H. \& Sîrbu, M., Optimal Investment on Finite Horizon with Random Discrete Order Flow in Illiquid Markets

Gatheral, J. \& Schied, A., Optimal Trade Execution Under Geometric Brownian Motion in the Almgren and Chriss Framework

Glasserman, P. \& Wu, Q., Forward and Future Implied Volatility

Grasselli, M., see Da Fonseca, J.

Guhr, T., see Münnix, M. C. Hadjiliadis, O., see Carr, P. Hillairet, C. \& Jiao, Y., Information Asymmetry in Pricing of Credit Derivatives
4 (2011) 507

5 (2011) 669

7 (2011) 1139

4 (2011) 559

2 (2011) 197

1 (2011) 41

1 (2011) 163

1 (2011) 1

4 (2011) 433

2 (2011) 265

1 (2011) 17

3 (2011) 353

3 (2011) 407

6 (2011) 899

8 (2011) 1231

8 (2011) 1195

5 (2011) 611
Ielpo, F., see Da Fonseca, J. Imkeller, P., Réveillac, A. \& Zhang, J., Solvability and Numerical Simulation of BSDEs Related to BSPDEs with Applications to Utility Maximization

Ishida, I., see Fukasawa, M.

Janson, S., M'Baye, S. \& Protter, P., Absolutely Continuous Compensators Janssen, J., see D'Amico, G. Jiao, Y., see Hillairet, C.

Joshi, M. S., see Fries, C. P.

Jourdain, B., Lapeyre, B. \& Sabino, P., Convenient Multiple Directions of Stratification

6 (2011) 867

5 (2011) 723

Kohlmann, M., see Xiong, D.

Korn, O. \& Koziol, P., The Term Structure of Currency Hedge Ratios

Koziol, C., Proelss, J. \& Schweizer, D., Do Institutional Investors Care About the Ambiguity of Their Assets? Evidence from Portfolio Holdings in Alternative Investments

Koziol, P., see Korn, O.

Kulperger, R., see Badescu, A.

Kupper, M., see Cheridito, P. Lapeyre, B., see Jourdain, B.

Lari-Lavassani, A., see Dmitrašinović-Vidović, $\mathrm{G}$.

Levendorskii, $\quad$ S., see Boyarchenko, M.

Li, X., see DmitrašinovićVidović, G.

Lo, H. \& Mijatović, A., Volatility Derivatives in Market Models with Jumps

Løkka, A. \& Zervos, M., A Model for the Long-Term Optimal Capacity Level of an Investment Project

Lorenz, S., see Dimitroff, G.

Lorig, M., Time-Changed Fast Mean-Reverting Stochastic Volatility Models

5 (2011) 635

4 (2011) 433

3 (2011) 335

2 (2011) 221

5 (2011) 611

2 (2011) 197

$4(2011) 525$

4 (2011) 465

4 (2011) 525

5 (2011) 669

1 (2011) 137

6 (2011) 867

6 (2011) 957

7 (2011) 1045

6 (2011) 957

7 (2011) 1159

2 (2011) 187

8 (2011) 1299

8 (2011) 1355

Madan, D. B. \& Schoutens, W., Conic Finance and the Corporate Balance Sheet

Maggis, M., see Frittelli, M.

Maghrebi, N., see Fukasawa, M.

Manca, R., see D'Amico, G.
5 (2011) 587

1 (2011) 41

4 (2011) 433

2 (2011) 221 
Marabel, J., Pricing Digital Outperformance Options with Uncertain Correlation

Mavroudis, K. \& Nolder, C. A., Fixed-Mix Rules in an Evolutionary Market Using a Factor Model for Dividends

M'Baye, S., see Janson, S.

Mercuri, L., Pricing Asian Options in Affine Garch Models

Miettinen, J., see Badescu, A.

Mijatović, A., see Lo, H.

Monoyios, M. \& Ng, A., Optimal Exercise of an Executive Stock Option by an Insider

Musiela, M. \& Zariphopoulou, T., Initial Investment Choice and Optimal Future Allocations Under TimeMonotone Performance Criteria

Münnix, M. C., Schäfer, R. \& Guhr, T., Statistical Causes for the Epps Effect in Microstructure Noise

Nadtochiy, S., see Carmona, R.

$\mathrm{Ng}$, A., see Monoyios, M.

Nolder, C. A., see Mavroudis, K.

Ohsaki, S. \& Yamazaki, A., Static Hedging of Defaultable Contingent Claims: A Simple Hedging Scheme Across Equity and Credit Markets

Oya, K., see Fukasawa, M.

Pakkanen, M. S., Brownian Semistationary Processes and Conditional Full Support

Pallavicini, A., see Brigo, D.

Papatheodorou, V., see Brigo, D.

Penner, I., see Föllmer, H.

Pham, H., see Gassiat, P.

Pistorius, M., see Eriksson, B.

Proelss, J., see Koziol, C.

Protter, P., see Janson, S.

Réveillac, A., see Imkeller, P.

Rodríguez, J. F., Hedging Swing Options

Rosazza Gianin, E., see Frittelli, M.
5 (2011) 709

8 (2011) 1247

3 (2011) 335

2 (2011) 313

5 (2011) 669

7 (2011) 1159

1 (2011) 83

1 (2011) 61

8 (2011) 1231

1 (2011) 107

1 (2011) 83

8 (2011) 1247

2 (2011) 239

4 (2011) 433
4 (2011) 579
6 (2011) 773
6 (2011) 773
1 (2011) 1
1 (2011) 17
7 (2011) 1139
4 (2011) 465
3 (2011) 335
5 (2011) 635
2 (2011) 295
1 (2011) 163

Sabino, P., see Jourdain, B.

Schäfer, R., see Münnix, M.

$$
\text { C. }
$$

6 (2011) 867

8 (2011) 1231

3 (2011) 353

5 (2011) 587

4 (2011) 465

5 (2011) 757

1 (2011) 17

5 (2011) 669

3 (2011) 369

8 (2011) 1299 G.

Takahashi, A., Tsuzuki, Y. \& Yamazaki, A., Hedging European Derivatives with the Polynomial Variance Swap Under Uncertain Volatility Environments

Tang, D., Wang, Y. \& Zhou, Y., Counterparty Risk for Credit Default Swap with States Related Default Intensity Processes

Tankov, P., see Brodén, M.

Tsuzuki, Y., see Takahashi, A.

Ubukata, M., see Fukasawa, M.

Wang, Y., see Bo, L.

Wang, Y., see Tang, D.

Ware, A., see DmitrašinovićVidović, G.

Wu, Q., see Glasserman, P.

Xiong, D. \& Kohlmann, M., The Compatible BondStock Market with Jumps Yamazaki, A., see Ohsaki, S.

Yamazaki, A., see Takahashi, A.

Yamazaki, K., see Fukasawa, M.

Yang, X., see Bo, L.

Yi, C., Dangerous Knowledge: Credit Value Adjustment with Credit Triggers

Zariphopoulou, T., see Musiela, M.

Zervos, M., see Løkka, A.

Zhang, H., see Carr, P.

Zhang, J., see Imkeller, P.

Zhou, Y., see Tang, D.

Zhu, S.-P. \& Chen, W.T., Should an American Option be Exercised Earlier or Later if Volatility is not Assumed to be a Constant?
4 (2011) 485

8 (2011) 1335

6 (2011) 803

4 (2011) 485

4 (2011) 433

6 (2011) 945

8 (2011) 1335

6 (2011) 957

3 (2011) 407

5 (2011) 723

2 (2011) 239

4 (2011) 485

4 (2011) 433

6 (2011) 945

6 (2011) 839

1 (2011) 61

2 (2011) 187

8 (2011) 1195

5 (2011) 635

8 (2011) 1335

8 (2011) 1279 